\title{
Des Flux
}

\section{Didier Josselin}

\section{OpenEdition}

\section{Journals}

Édition électronique

URL : http://journals.openedition.org/rge/4265

DOI : $10.4000 /$ rge.4265

ISSN : 2108-6478

\section{Éditeur}

Association des géographes de l'Est

\section{Édition imprimée}

Date de publication : 1 septembre 1999

ISSN : 0035-3213

\section{Référence électronique}

Didier Josselin, « Des Flux », Revue Géographique de l'Est [En ligne], vol. 39 / 4 | 1999, mis en ligne le 12 août 2013, consulté le 25 septembre 2020. URL : http://journals.openedition.org/rge/4265 ; DOI : https://doi.org/10.4000/rge.4265

Ce document a été généré automatiquement le 25 septembre 2020

Tous droits réservés 


\title{
Des Flux
}

\author{
Didier Josselin
}

1 Cet éditorial s'inspire d'une réflexion épistémologique que les géographes ont initiée il y a quelques années déjà ${ }^{1}$ et qui se nourrit des apports des sciences de la vie et la physique. Dans le fil de cette réflexion, certaines analogies et métaphores nous aiderons à faire image pour illustrer notre propos.

2 La démarche consiste en fait à expliciter, à travers des exemples de la Nature anthropisée, les différentes raisons qui m'ont poussé à éditer, avec l'aide des collègues de Besançon du laboratoire THEMA, un numéro de la Revue Géographique de l'Est sur

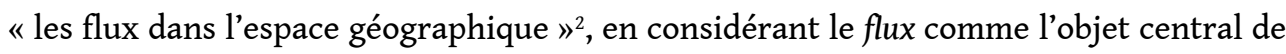
recherche.

\section{Au commencement, étaient les flux...3}

3 Il y a une quinzaine de milliards d'années, selon la loi de Hubble, au terme d'une brutale et immense explosion, le big bang, l'Univers aurait été créé.

4 Constitué de neutrons, de protons et de leptons en interaction, l'Univers connaît alors des températures très élevées. D'après le biochimiste soviétique Oparin et le biologiste anglais Haldane, l'atmosphère primitive de la Terre se compose d'hydrogène, de méthane, d'ammoniac et de vapeur d'eau. Soumis à de fortes chaleurs et au rayonnement du Soleil, cette « soupe primitive » chaude, tombant au fond des océans, aurait engendré des molécules organiques, puis les premiers organismes vivants.

5 En réalisant des simulations en laboratoire, les chercheurs ont observé la formation d' agrégats complexes, nommés "coacervats", "microsphères" ou "protobiontes ", véritables précurseurs des cellules vivantes, entourés d'une sorte de membrane rudimentaire permettant les échanges avec le milieu.

6 Par la suite, la recherche en physique a clairement identifié la notion de chaleur comme la manifestation d'un flux d'échange énergétique entre des éléments matériels contigus.

7 En 1969, Prigogine définit le concept de "structure dissipative », selon lequel, dans des systèmes traversés par des flux de matière et d'énergie, peuvent se produire des 
processus de structuration et d'organisation spontanées. Cette découverte vient contrecarrer un des principes de la thermodynamique, qui pose que les processus producteurs d'entropie produisent généralement une évolution irréversible d'un système vers un état de désordre maximal. En réalité, pour le "vivant ", un ensemble de réactions métaboliques, sous certaines conditions de flux, peut produire un (ou des) régime(s) collectif(s) au fonctionnement stable. L'irréversibilité, loin de l'équilibre théorique, peut jouer un rôle constructif et devenir source d'ordre. C'est «l'entropie négative » ou « néguentropie ».

Des flux de matière et d'énergie sont ainsi à la base de l'organisation de notre environnement.

\section{Autour de nous, les flux différencient, polarisent, organisent...}

9 Notre environnement direct, quelle que soit l'échelle à laquelle nous l'observons, offre de nombreux exemples où les flux continuent de fonctionner et de structurer le monde.

Migration, mouvement, transport sont nos premiers mots-clés.

11 Dans les organismes vivants, au sein des neurones, une pompe (dite "pompe à sodium ») transporte en permanence des ions à travers la membrane cellulaire. Les pays et les villes connaissent des processus bien connus d'immigration et d'émigration, associés à des transports de matières et d'informations. Des flux d'énergie au sein des dorsales océaniques provoquent la dérive des continents. Qu'il s'agisse de mouvements incessants d'ions à travers les membranes cellulaires, de migrations alternantes des populations actives ou de déplacements d'énergie dans la croûte océanique, il règne une dynamique forte dans l'ensemble des systèmes présents sur la Terre, qu'ils soient naturels ou anthropiques.

Les flux incarnent cette dynamique et les différentes formes qu'elle peut revêtir.

13 Cette dynamique est à l'origine des phénomènes de différenciation, de polarisation ou d' agrégation.

14 Ce n'est pas un hasard (quoique...) si, après les agrégats de la "soupe primitive ", l'on parle de différenciation cellulaire, en évoquant la constitution d'une cellule porteuse d'une information à la fois unique et analogue à celle d'autres cellules (paradoxe de la reproductibilité et de l'unité de la vie). Si cette cellule est un neurone, la pompe à ions crée une polarisation permanente, polarisation que seul un influx nerveux pourra inverser, déclenchant ainsi un autre type de flux : un potentiel d'action, porteur d'une information sensitive. Potentiel, différentiel ou tension (au sens de la différence entre deux valeurs en deux points), sont des notions proches. Dans le cas précis des dorsales océaniques, les tensions peuvent provoquer des ruptures, se concrétisant en éruptions volcaniques, raz de marée ou séismes.

15 L'analogie avec les espaces géographiques est loin d'être dénuée de sens. On parle parfois d'osmose entre pays industrialisés et en voie de développement, mouvement renforcé par les flux de capitaux. De façon similaire, les capitales régionales, génératrices d'emplois, deviennent attractives, au détriment de bassins déprimés, provoquant l'agglomération des populations et une polarisation de l'espace géographique. 
16 Ainsi, les flux sont à la fois sources de différenciation de notre environnement et potentiellement déclencheurs d'évènements physiques, de "ruptures » ou de "fractures » physiques, politiques ou sociales.

Enfin, il semble que le simple fait d'observer l'existence des processus ne suffise pas pour bien les appréhender. Il paraît nécessaire de les mesurer et de les qualifier.

De fait, la quantité d'ions présente de part et d'autre de la membrane cellulaire neuronale est directement liée au potentiel d'action, le nombre de navetteurs journaliers indique la force de l'attractivité d'une ville ou du lien qui unit les deux villes concernées, les masses de matières émergeant des dorsales poussent les plaques océaniques, qui contraignent plus ou moins les zones de subduction.

19 Respectivement, la qualification des éléments composant les flux doit être prise en compte. De quel type d'ions la pompe transmembranaire se charge-t-elle? Quelles sont les caractéristiques sociales des navetteurs? Quels sont les trajectoires et les mouvements tectoniques des plaques les unes par rapport aux autres?

Dans une approche de type systémique, la complémentarité entre la quantification des flux et la sémantique qui leur est associée, peut permettre une meilleure compréhension des phénomènes, comme ceux qui régissent l'espace géographique.

\section{La problématique des flux au cœur des préoccupations du laboratoire THEMA}

21 Considérer les flux comme ubiquistes et toujours à la base des processus et de l'organisation de notre environnement peut, je le conçois, paraître réducteur. Les structures ne conditionnent-elles pas les flux ? Répondre non à cette question serait, à l'évidence, nier toutes les boucles de rétroaction et les interactions entre les composants complexes des systèmes. Pourtant, en lançant l'idée d'un numéro spécial sur les « flux dans l'espace géographique ", j'avais, et j'ai toujours, l'intime conviction que le flux revêt une spécificité et un intérêt forts.

Outre sa propre sémantique, le flux porte une partie de l'information associée aux entités géographiques qu'il relie. En ce sens, il est plus qu'un simple objet d'analyse, puisqu'il intègre une part de la structure avec laquelle il est en relation. Il fournit ainsi un éclairage différent sur les structures visibles. De plus, pour des raisons de disponibilité et de mesurabilité de l'information, il est commun et, en général commode, d'étudier les formes du monde visible, plutôt que leurs interactions. Prendre le flux comme la référence d'analyse constitue donc une approche originale. Enfin, rien n'interdit, comme les lecteurs le noteront à travers les articles de ce numéro, d'aborder, même sous l'angle des flux, la dialectique entre ceux-ci et les structures, cette approche renforçant d'autant l'analyse spatiale.

Le flux est donc un point de vue, objectif car identifiable, subjectif car orienté, sur les processus et des dynamiques dans l'espace géographique.

24 Une seconde justification de ce numéro est la spécificité du laboratoire THEMA dans le domaine en question. Il est rare de trouver, au sein d'une même équipe de recherche, une telle variété d'approches des flux (flux de véhicules, de personnes, de matière et d'information) qui mêlent, à divers degrés, thématique et méthodologie. 
mier ensemble d'articles concerne les flux de circulation routière. P. Bérion et $\mathrm{D}$. Mathieu présentent deux analyses complémentaires de l'observatoire de l'autoroute A39, C. Genre-Grandpierre étudie les réseaux sous les angles structurel et fonctionnel, A. Banos aborde le problème des flux transalpins de poids-lourds.

La Suisse, plus précisément l'arc jurassien franco-suisse, est également un terrain d'analyse privilégié des migrations (A. Moine). M. Griselin et S. Ormaux nous parlent des « hub and spokes » et de leur place dans les transports aériens de personnes. Enfin, les flux touchent aussi à des mouvements de matière et d'information. Les articles de C. Janin et moi-même sur les flux agricoles intercommunaux et l'analyse des flux téléphoniques réalisée par J.-C. Chevailler et P. Gillon en sont deux exemples.

L'ensemble de ces articles ne prétend pas "faire le tour de la question». Il constitue cependant un florilège de thèmes et des méthodes variés que je vous invite à découvrir, dans ce numéro sur "les flux dans l'espace géographique ».

\section{NOTES}

1. Voir par exemple, les réflexions du Groupe Dupont, dans le Géopoint de 1984, notamment l'article d'A. Dauphiné "systémogenèse, théorie biologique et géographie »; les ouvrages qui abordent l'histoire de la géographie, comme « La face de la terre, éléments de géographie » de P. et G. Pinchemel, Colin, 1992 ; J. Sheibling, «Qu'est-ce que la géographie ? ", Carré Géographie, Hachette Supérieur, 1994; ainsi que des recueils d'articles et de définitions, comme l'« Encyclopédie de Géographie » d'A. Bailly, R. Ferras, D. Pumain, Economica, 1992 ; R.-J. Johnson, D. Gregory, D.-M. Smith, «The dictionnary of human geography », Backwell Reference, 1994 ; A. Goudie et al., « The Encyclopedic dictionnary of physical geography », Backwell Reference, 1994.

2. Je remercie notamment T. Brossard, H. Chamussy, J.-C. Foltête, M. Griselin, A.-M. Odouze et S. Ormaux pour leurs relectures et leurs conseils.

3. Ce paragraphe s'est inspiré des articles de l'Encyclopedia Universalis; de l'ouvrage «La cellule », Biologie moléculaire, de Darnell, Lodish, Baltimore, Editions Décarie-Vigot, 1988; de l'« Atlas de poche de Physiologie » de S. Sibernagl et A. Despopoulos, Flammarion, 1986.

\section{AUTEUR \\ DIDIER JOSSELIN}

Laboratoire THEMA - Université de Franche-Comté - 32, rue Mégevand 25030 Besançon Cedex 